\title{
Thanks to our Reviewers of the CEAS Space Journal
}

\section{(C) CEAS 2017}

It is well-known that the quality of a scientific/technical journal strongly depends on the rigorous work of the people involved in the review process, i.e. the field editors, but in particular the reviewers.

Hence, the editors of the CEAS Space Journal highly appreciate the dedication and the contributions of the reviewers listed below, who have helped to maintain and improve the quality of the journal in 2016 .

$\begin{array}{lll}\text { S. Appel } & \text { J. Harder } & \text { G. Saccoccia } \\ \text { H. Baier } & \text { A. Heuberger } & \text { F. Schrijer } \\ \text { P. Barbante } & \text { P. Hodgetts } & \text { R. Schwane } \\ \text { H. Barde } & \text { A. Ihle } & \text { L. Scialino } \\ \text { S. Bennani } & \text { R. Jensen } & \text { J. Shan } \\ \text { H. Bischl } & \text { A. Kolesnikov } & \text { A. Siegmund } \\ \text { C. Bonnal } & \text { U. Lafont } & \text { V. Simard Bilodeau } \\ \text { O. Bozic } & \text { M. Landgraf } & \text { M. Sippel } \\ \text { S. Chesne } & \text { R. Laufer } & \text { M. Tajmar } \\ \text { G. Colonna } & \text { R. Lecourt } & \text { S. Theil } \\ \text { A. Corbelli } & \text { H. Leiter } & \text { J. Thoemel } \\ \text { J. Dalsgaard Nielsen } & \text { S. Löhle } & \text { E. Trifoni } \\ \text { R. Degenhardt } & \text { E. Lorenz } & \text { J. Vandersteen } \\ \text { V. Drakonakis } & \text { H. Lüdeke } & \text { E. Vanhove } \\ \text { O. Dubois-Matra } & \text { E. Mair } & \text { S. Voigt } \\ \text { S. Ducruix } & \text { V. Maiwald } & \text { T. Voirin } \\ \text { C. Dupuy } & \text { M. Marini } & \text { F. Wagner } \\ \text { C. Elia } & \text { A. Martinez Barrio } & \text { B. Wie } \\ \text { S. Erb } & \text { J. Merrifield } & \text { E. Wille } \\ \text { A. Eriksson } & \text { E. Mooij } & \text { M. Winter } \\ \text { M. Falkner } & \text { F. Nasuti } & \text { K. Wormnes } \\ \text { E. Farbar } & \text { L. Newman } & \text { C. Yang } \\ \text { M. Fertig } & \text { I. Ngan } & \\ \text { B. Fritsche } & \text { A. Nicole } & \\ \text { M. A. Gallis } & \text { G. Ortega } & \\ \text { J. Gil Fernandez } & \text { M. Panesi } & \\ \text { J. Gomez Garcia } & \text { D. Prabhu } & \\ \text { M. Grabe } & \text { P. Rebeyre } & \\ \text { P. Grenard } & \text { A. Rinalducci } & \\ & & \end{array}$

